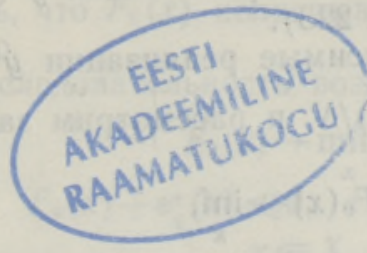

\title{
СТАТИСТИЧЕСКОЕ ИССЛЕДОВАНИЕ И МЕТОД РЕШЕНИЯ ЗАДАЧ СТОХАСТИЧЕСКОГО ПРОГРАММИРОВАНИЯ
}

Получены условия, при которых решение нелинейной задачи стохастического программирования с ограничениями на средние значения есть предел с вероятностью 1 решений задач, в которых ограничения на средние значения заменены ограничениями на их статистические оценки. Тем самым предложен метод приближенного решения исходной задачи по статистическим данным. Более подробно рассмотрено решение по средним значениям задачи линейного программирования, в которой только правые части случайные.

1. Расомотрим задачу стохастического іпрограммирования

$$
\begin{gathered}
F_{0}(x)=M f_{0}(x, y) \rightarrow \inf _{x} \\
F_{i}(x)=M f_{i}(x, y) \leqslant 0, \quad i=1, \ldots, m, \\
x \in X \subset R^{n}, \quad y \in Y \subset R^{s},
\end{gathered}
$$

где $y-s$-мерная случайная величина, закон распределения которой не зависит от $x$. Задачи с вероятностными и квантильными ограничениями также могут быть записаны в виде (1)-(3).

Существует много работ (особенно при линейных функциях $\left.f_{i}(x, y)\right)$, где решение задачи $(1)-(3)$ рассматривается при заданном раопределении величины $y$ или некоторых ее моментов (см., напр., $\left.\left[{ }^{1-3}\right]\right)$. Но предположение о том, что закон распределения $y$ или моменты $y$ известны, слишком жесткое, при его выполнении задача в сущности становится детерминированной (см. $\left.\left[{ }^{4}\right]\right)$.

Применение прямых методов решения более перспективно с точки зрения приложения стохастического программирования. В прямых методах вместо закона распределения $y$ предполагаются известными реализации $y^{1}, y^{2}, \ldots, y^{N} \ldots$. Если же закон распределения $y$ задан, но найти интегралы $M f_{i}(x, y)$ аналитически не удается, определить их можно с помощью одного из численных методов интеприрования. В случае большой размерности пространства $R^{n}$ для вычисления интегралов более эффективен метод Монте-Карло. Следует сказать, что для решения (1) - (3) нет надобности вычислять $M f_{i}(x, y)$ в каждой точке $x$ точно. Оценки интегралов должны определять направление убывания $M f_{i}(x, y)$ только «в среднем». Исходя из этих соображений и пострбены прямые методы.

Прямые методы, в том числе и метод стохастической аппроксимации, сходятся медленно (как все методы, использующие идеи метода Монте-Карло). За ограниченное число реализаций можно оказаться далеко от истинного минимума, как и случилось в задаче, рассмотрен- 
ной в $\left[{ }^{5}\right]$. Поэтому представляет интерес разработка таких прямых методов, которые бы позволили приближенно решить задачу (1)-(3) и при сравнительно небольшом числе реализаций $y^{k}(k=1, \ldots, N)$. То же самое отмечается и в $\left[{ }^{4}\right]$ (с. 223).

Итак, пусть заданы независимые реализации $y^{1}, y^{2}, \ldots, y^{N}$. Обозначим $\hat{F}_{i}(x, y, N)=\sum_{k=1}^{N} f_{i}\left(x, y^{k}\right) / N$ и рассмотрим задачу

$$
\begin{gathered}
F_{0}(x) \rightarrow \underset{x}{\inf ,} \\
\hat{F_{i}}(x, y, N)=\frac{\sum_{k=1}^{N} f_{i}\left(x, y^{k}\right)}{N} \leqslant 0, \quad i=1, \ldots, m, \\
x \in X .
\end{gathered}
$$

Можно считать, что функция $F_{0}(x)$ задана в аналитическом виде, в противном случае ее можно заменить дополнительной переменной $x_{n+1}$. Если (1)-(3) задача вероятностной природы, то (4)-(6) задача статистической природы. Вместо решения (1)-(3) мы получим некоторую его оценку $\hat{x}(N)$ (решенне задачи $(4)-(6))$, которая зависит от реализаций $y^{k}(k=1, \ldots, N)$.

Допустим, что задача (1)-(3) имеет решение $x^{*}$. У задачи (4)(6) решения может и не быть (есть примеры, когда с вероятностью 1 решение задачи (4)-(6) не существует). Если при некотором $N$ и реализациях $y^{1}, y^{2}, \ldots, y^{N}$ решение $\hat{x}(N)$ не существует, то для определенности положим $\hat{x}(N)=0$ (условиями последующих теорем 3 и 4 гарантируется стремление к 1 при $N \rightarrow \infty$ вероятности того, что задача (4)-(6) имеет решение). Отметим, что в теоремах 3 и 4 не имеет значения, чему равняется $\hat{x}(N)$ в том случае, когда решение (4) - (6) в действительности не существует.

Обозначим через $Q$ и $Q(N)$ множества допустимых решений задач (1) - (3) и (4)-(6) соответственно.

Поскольку оценка $\hat{x}(N)=\hat{x}\left(N, y^{1}, \ldots, y^{N}\right)$ является случайной величиной, возникают обычные проблемы статистического оценивания проблемы состоятельности, несмещенности и эффективности оценки $\hat{x}(N)$. Часть из них будет рассмотрена в дальнейшем в предположении, что все рассматриваемые здесь случайные величины измеримы.

2. Вопрос о том, можно ли задачу (1)-(3) заменить задачей (4)(6) и тем самым получить в некотором смысле хорошую оценку $x$ * через $\hat{x}(N)$, во многом совпадает с проблемой непрерывности решения задачи нелинейного программирования при возмущении ограничений. Эта проблема имеет много аспектов, для нашей задачи важно то, что по терминологии $\left[{ }^{6}\right]$ называется вертикалыной устойчивостью.

Рассмотрим детерминированную задачу

$$
\begin{gathered}
F_{0}(x) \rightarrow \min _{x}, \\
F_{i}(x) \leqslant 0, \quad i=1,2, \ldots, m, \\
x \in X
\end{gathered}
$$

и назовем ее невозмущенной.

Допустим, что $X$ - компакт в $R^{n}, F_{0}(x)$ - непрерывная функция и ее минимум в $Q$ достигается в единственной точке $x^{*}:$ Если $F_{0}(x)$ раз- 
рывна, то это значительно усложняет задачу (см. [7]). Путем введения дополнительной переменной и дополнительного ограничения всегда можно добиться непрерывности целевой функции. Поэтому в дальнейшем будем считать, что $F_{0}(x)$ непрерывная функция, значения которой вычисляются точно.

Рассмотрим последовательность возмущенных задач

$$
\begin{gathered}
F_{0}(x) \rightarrow \min _{x}, \\
F_{i}(x)+\varepsilon_{i}^{s}(x) \leqslant 0, \quad i=1, \ldots, m, \\
x \in X,
\end{gathered}
$$

где $\left\{\varepsilon_{i}^{s}(x)\right\}, s=1,2, \ldots$, последовательность непрерывных функций в $X, i=1, \ldots, m$. Пусть $\varepsilon_{i}^{s}(x) \rightarrow 0, \quad s \rightarrow \infty$, для всех $x \in X$ и $i=1, \ldots, m$. Решение задачи $(10)-(12)$, вообще говоря, не единственно. Обозначим через $x^{*}\left(\varepsilon^{s}\right)$ любое из возможных ее оптимальных решений при фиксированном $\delta$.

О п р еделен и е 1. Задача (7)-(9) называется вертикально устойчивой, если $x^{*}\left(\varepsilon^{s}\right) \rightarrow x^{*}, s \rightarrow \infty$, для любой последовательности равностепенно непрерывных функций $\left\{\varepsilon_{i}^{s}(x)\right\}, \varepsilon_{i}^{s}(x) \rightarrow 0, \quad s \rightarrow \infty, i=$ $=1, \ldots, m$.

Чтобы уяснить характер условий, которые нужно наложить на $F_{i}(x)$, рассмотрим пример (рисунок).

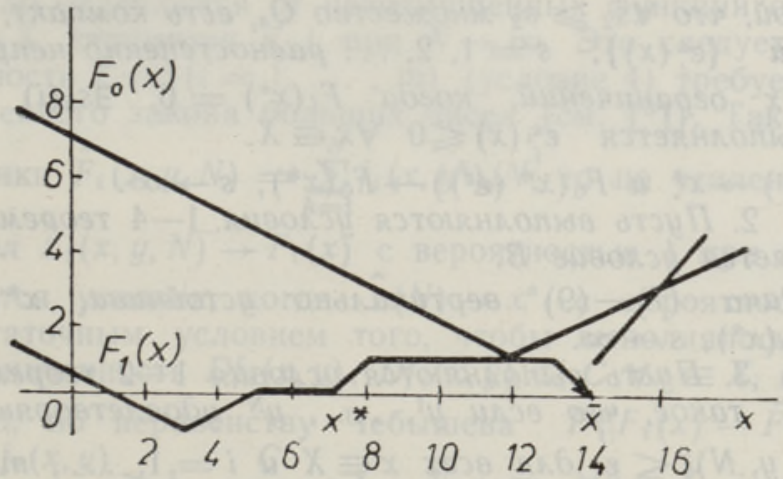

Если $X=[0,16]$, то $Q=[1,7]$. При отрицательном вертикальном возмущении $F_{1}(x)$, если ограничение $F_{1}(x) \leqslant 0$ заменить ограничением $F_{1}(x) \leqslant 0$, где $F_{1}(x)<F_{1}(x), x \in X$, для любого малого возмущения точка минимума «перескакивает» из точки $x^{*}=7$ в окрестность точки $\bar{x}=14$. Это означает, что при расширении множества допустимых решений точка минимума изменяется разрывно. В дальнейшем такое изменение запрещается условием $A$.

При положительном вертикальном возмущении, $\tilde{F}_{1}(x)>F_{1}(x)$, точка минимума скачком переходит в окрестность точки $x=5$. В дальнейшем это запрещается условием $B$.

Как видно из примера, даже если все функции $F_{i}(x)$ непрерывны (напр., $X=[0,10])$, точка минимума меняется разрывно.

У сл ов не $A$. Пусть $x_{k} \in X \backslash Q, \quad x_{k} \rightarrow \bar{x}, \quad F_{0}\left(x_{k}\right) \rightarrow a_{0}, k \rightarrow \infty$. Eсли $\lim F_{i}\left(x_{k}\right) \leqslant 0$ для всех $i=1, \ldots, m$, то $F_{0}(\bar{x})>F_{0}\left(x^{*}\right)$.

Условие $A$ обеспечивает непрерывное изменение точки минимума при расширении множества допустимых решений (при вертикальном возмущении ограничений). Для непрерывных $F_{i}(x)(i=0,1, \ldots, m)$ в случае единственной точки минимума условие $A$ выполняется (тогда 
$F_{i}(\bar{x}) \leqslant 0, i=1, \ldots, m$, и $\bar{x} \in Q$, а $F_{0}(\bar{x})>F_{0}\left(x^{*}\right)$ в силу единственности $\left.x^{*}\right)$.

Услови е $B$. Пусть $U=\left\{x: x \in X, F_{i}(x)<0, \quad i=1, \ldots, m\right\}$. Замыкание $U$ содержит точку $x^{*}, x^{*} \in \bar{U}$.

Условие $B$ обеспечивает непрерывное изменение точки минимума при сужении множества допустимых решений (при вертикальном возмущении ограничений). Очевидно, что из условия $B$ следует существование числа $k_{0}$ и последовательности $x_{k} \in Q, x_{k} \rightarrow x^{*}, k \rightarrow \infty, x_{k} \neq x^{*}$ таких, џто $\forall k \geqslant k_{0} F_{i}\left(x_{k}\right)<0$ для всех $i=1, \ldots, m$.

3. Приведем некоторые утверждения и пояснения к ним. Для краткости теоремы даются без доказательств.

Л е м м а 1. Предположим, ито 1) если функции $F_{i}(x)(i=1, \ldots, m)$ амеют точки разрыва, то их конечное число. Пусть $c_{i j}, j=1, \ldots, r_{i}$, точки разрьва, причем если $F_{i}\left(c_{i j}\right)>0$, то и $\lim F_{i}\left(x_{k}\right)>0, x_{k} \rightarrow c_{i j}$, $k \rightarrow \infty, \forall j=1, \ldots, r_{i}, i=1, \ldots, m$.

2) если $F_{i}(c)=0$, то $\lim F_{i}\left(x_{k}\right) \geqslant 0, x_{k} \rightarrow c, k \rightarrow \infty$.

Тогда, во-первых, множсесто $Q$ есть компакт, $u$, во-вторых, $\exists s_{1} т а$ кой, что $\forall s_{2} \geqslant s_{1}$ множество $Q_{s_{2}}$ есть компакт.

Т е ор ем а 1. Пусть выполняются условия:

1) множество $X$ - компакт в $R^{n}$, a $x^{*}$ единственная точка минимума непрерьвной функции $F_{0}(x)$ в $Q$,

2) условие $A$ выполняется,

3) $\exists s_{1}$ такой, что $\forall s_{2} \geqslant s_{2}$ множество $Q_{s_{2}}$ есть компакт,

4) функции $\left\{\varepsilon_{i}^{8}(x)\right\}, s=1,2, \ldots$ равностепенно непрерывны в $X$,

5) для тех ограничений, когда $F_{i}\left(x^{*}\right)=0, \exists s_{3}(i)$ такой, что $\forall s_{4} \geqslant s_{3}(i)$ выполняется $\varepsilon_{i}^{s_{4}}(x) \leqslant 0 \quad \forall x \in X$.

Тогда $x^{*}\left(\varepsilon^{s}\right) \rightarrow x^{*} u F_{0}\left(x^{*}\left(\varepsilon^{s}\right)\right) \rightarrow F_{0}\left(x^{*}\right), s \rightarrow \infty$.

Те орем а 2. Пусть выполняются условия 1-4 теоремы 1 и

5) выполняется условие $B$.

Тогда задача (7)-(9) вертикально устойчива, $x^{*}\left(\varepsilon^{s}\right) \rightarrow x^{*}$ и $F_{0}\left(x^{*}\left(\varepsilon^{s}\right)\right) \rightarrow F_{0}\left(x^{*}\right), s \rightarrow \infty$.

Т еорем а 3. Пусть выполняются условия $1-2$ теоремы $1 ~ u$

3) $\exists \varepsilon_{0}>0$ такое, что если $y^{1}, \ldots, y^{N}$ удовлетворяют условиям $\left|F_{i}(x)-\hat{F}_{i}(x, y, N)\right|<\varepsilon$ для всех $x \in X \quad u i=1, \ldots, m$, то множество допустимых решений $\hat{Q}(N)$ задачи (4)-(6) есть компакт для любого $\varepsilon, 0<\varepsilon \leqslant \varepsilon_{0}$,

4) функции $F_{i}(x)(i=1, \ldots, m)$ ограничены в $X$,

5) выполняется условие $B$,

6) $\forall \varepsilon>0$ вероятность $P\left\{\left|F_{i}(x)-\hat{F}_{i}(x, y, N)\right|<\varepsilon\right\} \rightarrow 1$ равномерно по всем $x \in X, N \rightarrow \infty, i=1, \ldots, m$.

Тогда $\hat{x}(N) \rightarrow x^{*}$ и $F_{0}(\hat{x}(N)) \rightarrow F_{0}\left(x^{*}\right)$ с вероятностью 1 при $N \rightarrow \infty$.

Т ео рем а 4. Пусть выполняются условия

1) $x^{*}$ единственная точка минимума сx в $Q=\left\{x: M a_{i} x-M b_{i} \leqslant\right.$ $\left.\leqslant 0, i=1, \ldots, m, x_{k} \geqslant 0, k=1, \ldots, n\right\}$, где $\left|M a_{i j}\right|<\infty,\left|M b_{i}\right|<\infty$, $i=1, \ldots, m, j=1, \ldots, n$;

2) множество $Q$ имеет внутреннюю точку.

Тогда $\hat{x}(N) \rightarrow x^{*}$ u $\hat{x}(N) \rightarrow c x^{*}$ с вероятностью $1 n$ ри $N \rightarrow \infty$.

В лемме 1 даются простые достаточные условия компактности $Q$ (множества допустимых решений $(7)-(9))$ и $Q_{s}$ (множества допустимых решений $(10)-(12))$. Огевидно, условие 1 леммы 1 обеспечивает компактность $Q$, а условие 2 - компактность $Q_{s}$ при достаточно малом возмущенин. 
В теоремах 1 и 2 условие компактности (условие 3) предполагается выполненным. Равномерная непрерывность $\left\{\varepsilon_{i}^{s}(x)\right\}$ гарантирует, что $\forall \varepsilon>0$ найдется $s_{i}$ такой, что $\forall s \geqslant s_{i}$ выполняется $\mid F_{i}(x)-\left(F_{i}(x)+\right.$ $\left.+\varepsilon_{i}^{s}(x)\right) \mid<\varepsilon \forall x \in X$. В теореме 1 не предполагается выполненным условие $B$, но по условию 5 при достаточно больших $s$ выполняется включение $Q_{i} \subset Q_{\text {is }}$ для активных ограничений, а это означает, что непрерывное изменение точки минимума обеспечивается только при расширении множества допустимых решений. Последнее имеет место, так как выполняется условие $A$ (условие 2 ).

В теореме 2 допускается и сужение множества допустимых решений, но тогда непрерывность точки минимума обеспечивается условием $B$.

Если невозмущенная задача (7)-(9) имеет единственное решение $x^{*}$ и если $F_{i}(x) \quad(i=0,1, \ldots, m)$ непрерывны в компакте $X$, то условия $1-3$ теорем 1 и 2 выполняются.

Если решение невозмущенной задачи неединственно, то, вообще говоря, $x^{*}\left(\varepsilon^{s}\right)$ не сходится. В таком случае можно выделить подпоследовательность $x^{*}\left(\varepsilon^{s_{t}}\right)$, сходящуюся к любому из оптимальных решений.

Приступим к изучению статистической задачи (4)-(6). Рассмотрим условия теоремы 3. Условие 3 , как и в предыдущих теоремах, это требование компактности множества $Q(N)$ при достаточно малом возмущении. Вероятность того, что возмущенные функции ограничений $\hat{F}_{i}(x, y, N)$ мало отличаются от невозмущенных функций одновременно для всех $x \in X$, стремится к 1 при $N \rightarrow \infty$. Это следует из условия 6 . Ограниченность $F_{i}(x)(i=1, \ldots, m)$ (условие 4) требуется для применения усиленного закона больших чисел (см. $\left.\left[{ }^{8}\right]\right)$. Так как мы используем оценки $\hat{F}_{i}(x, y, N)=\sum_{k=1}^{N} f_{i}\left(x, y^{k}\right) / N$, то по усиленному закону больших чисел $\hat{F}_{i}(x, y, N) \rightarrow F_{i}(x)$ с вероятностью 1 при $N \rightarrow \infty$. Теоремой 3 даются условия, когда $\hat{x}(N) \rightarrow x^{*}$ с вероятностью 1 при $N \rightarrow \infty$. Достаточным условием того, чтобы выполнялось условие 6 , является ограниченность $D f_{i}(x, y), D f_{i}(x, y) \leqslant C \forall x \in X, i=1, \ldots, m$. Действительно, по неравенству Чебышева $P\left\{\left|F_{i}(x)-\hat{F}_{i}(x, y, N)\right|<\right.$ $<\varepsilon\} \geqslant 1-\frac{D f_{i}(x, y)}{N \varepsilon^{2}} \geqslant 1-\frac{C}{N \varepsilon^{2}} \rightarrow 1, \quad N \rightarrow \infty, \forall x \in X, i=1, \ldots, m$. Условие 6 , очевидно, выполняется, если $F_{i}(x)-f_{i}(x, y)$ не зависит от $x, i=1, \ldots, m$.

Рассмотрим линейную задачу

$$
\begin{gathered}
c x \rightarrow \min , \\
M a_{i} x \leqslant M b_{i}, i=1, \ldots, m, \\
x \in X=R_{+}^{n}=\left\{x: x_{k} \geqslant 0, k=1, \ldots, n\right\}, \\
c=\left(c_{1}, \ldots, c_{n}\right), a_{i}=\left(a_{i 1}, \ldots, a_{i n}\right), i=1, \ldots, m .
\end{gathered}
$$

Теорема 4 , в отличие от предыдущих теорем, доказывается для некомпактного множества $X=R_{+}^{n}$. Для применения усиленного закона больших чисел требуется конечность математических ожиданий коэффициентов. Условие $A$ для линейной задачи выполняется, а из того, что множество $Q$ имеет внутреннюю точку, следует, что выполняется и условие $B$.

Рассмотрим более подробно случай, когда только $b_{i}$ случайные величины. Последующие рассуждения в этом пункте носят неформаль- 
ный характер, строгие утверждения и формулировка понятий не приводятся. Допустим, что выполняются предположения теоремы 4 и пусть еще $Q$ - компакт. Для решения задачи (13)-(15) по средним $M b$ нужно, во-первых, определить базис, соответствующий оптимальному решению по средним $M b$ (б.с.о.р.ср.) (т. е. соответствующий $x^{*}$ ) и, вовторых, оценить $M b$ через $\hat{b}(N)$. Определение б.с.о.р.ср. заменим проверкой гипотезы о том, что среднее значение $M b m$-мерной случайной величины $b$ принадлежит области $D$ (это область, в которой при изменении правых частей, базис остается б.с.о.р.ср.). В работе [ $\left.{ }^{9}\right]$ область $D$ названа областью устойчивости, опраниченной $m$ пиперплоскостями. При нормировке уравнений этих гиперплоскостей и подстановке в них координат вектора $\hat{b}(N)$ получим расстояния от точки $\hat{b}(N)$ до граничных гиперплоскостей (расстояние от пиперплоскости в одном полупространстве будем считать положительным, в другом отрицательным). При решении задачи $(13)-(15)$ по оценке $\hat{b}(N)$ базис совпадает с б.с.о.р.ср., если расстояние от точки $\hat{b}(N)$ до всех граничных гиперплоскостей области устойчивости $D$ того же знака, что и от точки $M b$.

4. Рассмотрим задачу с вероятностными ограничениями

$$
\begin{gathered}
F_{0}(x) \rightarrow \underset{x}{\inf ,} \\
F_{i}(x)=p_{i}-P\left[\varphi_{i}(x, y) \leqslant 0\right] \leqslant 0, \quad i=1, \ldots, m, \\
x \in X .
\end{gathered}
$$

Положим

$$
f_{i}(x, y)= \begin{cases}p_{i}-1, & \text { если } \varphi_{i}(x, y) \leqslant 0 \\ p_{i}, & \text { если } \varphi_{i}(x, y)>0\end{cases}
$$

Тогда задача (16)-(18) с вероятностными ограничениями преобразуется в задачу с ограничениями на средние значения в виде (1)-(3).

Обозначим число реализаций $y^{k}(k=1, \ldots, N)$ таких, что $\varphi_{i}\left(x, y^{k}\right) \leqslant 0$, через $l_{i}(x), l_{i}(x)=\sum_{k: \varphi_{t}\left(x, y^{k}\right) \leqslant 0} 1$. Тогда ограничение (5) преобразуется к виду $p_{i}-l_{i}(x) / N \leqslant 0$ или к виду

$$
N p_{i} \leqslant l_{i}(x), \quad i=1, \ldots, m .
$$

Условия 4 и 6 теоремы 3 для задач с вероятностными ограничениями всегда выполняются. Если выполняются и остальные условия теоремы 3 , то имеет место сходимость $\hat{x}(N) \rightarrow x^{*}$ с вероятностью 1 при $N \rightarrow \infty$.

Следует добавить, что в задаче линейного пропраммирования со случайными коэффициентами $a_{i j}, b_{i}$, когда $\varphi_{i}(x, y)=\varphi_{i}\left(x, a_{i}, b_{i}\right)=$ $=a_{i 1} x_{1}+\ldots+a_{i n} x_{n}-b_{i}$, множество точек $x$, определяемое условием (19), в общем случае не является выпуклым.

Квантильная целевая функция может быть преобразована путем введения новой переменной в ограничение, а квантильные опраничения эквивалентны вероятностным.

5. Как правило, оценка $\hat{x}(N)$ смещена (если $M \hat{x}(N)$ существует). Несмещена она, например, в задаче линейного программирования $(13)-(15)$, когда только $b_{i}$ случайные величины. В условиях теоремы 3 имеет место асимптотическая несмещенность оценки $\hat{x}(N), \quad \hat{\hat{x}}(N) \rightarrow$ 
$\rightarrow x^{*}, N \rightarrow \infty$. Действительно, по теореме Лебега $\left[{ }^{4}\right]$ для ограниченных случайных величин из сходимости оценки с вероятностью 1 следует ее асимптотическая несмещенность.

Выражаю благодарность Т. Тобиасу за обсуждение работы.

\section{ЛИТЕ Р А Т Р А}

1. V a jda, S., Probabilistic programming, New York, London, 1972.

2. S e n g u pt a, J. K., Stochastic programming - methods and applications, Amsterdam, 1972 .

3. Юдин Д. Б., Математичеокие методы управления в условиях неполной информации, М., 1974.

4. Ермольев Ю. М., Методы стохастическопо пропраммирования, М., 1976.

5. Юби Э. А.-Ю., Тр. Таллинс. политехн. ин-та, № 411, 57 (1976).

6. Л ор ан П., Аппроксимация и оптимизация, М., 1975.

7. Eva n s, J. P., G o u ld, F. J., Oper. Res., 18, No. 1, 107 (1970).

8. Гнеденко Б. В., Курс тесрии вероятностей, М., 1961,

9. Арб уз ов а Н. Й., Экономика и математические методы, 2, № 4, 558 (1966).

\section{Таллинский политехнический} институт $\begin{array}{rl}\text { Поступила в редакцию } & \text { в } \\ 6 / \mathrm{I} & 1977\end{array}$

\section{E. $O B I$}

\section{STOHHASTILISE PLANEERIMISE ULESANNETE STATISTILINE UURIMINE JA LAHENDUSMEETOD}

Vaadeldakse stohhastilise planeerimise ülesannet, kus juhuslike suuruste keskväärtuste kitsendused on asendatud keskväärtuste hinnangute kitsendustega. Antakse tingimused, mille puhul lahendi hinnang koondub lähteülesande lahendiks.

\section{E. $O B I$}

\section{STATISTICAL INVESTIGATION OF STOCHASTIC PROGRAMMING PROBLEMS AND METHOD FOR THEIR SOLUTION}

The stochastic programming problem where the mean constraints are replaced by their estimates is investigated. The conditions under which the solutions of the problems with estimated means converge to the solutions of the original one are given. 